\title{
More than meets the eye- an unusual presentation of Cushing's syndrome with bilateral central retinal vein occlusion
}

The Royal Wolverhampton NHS Trust

EP46: Case Report- Pituitary

\section{Initial presentation}

- 53/M with H/O well-controlled hypertension, long-term smoking, alcohol excess, depression

- Presented to optician with intermittent blurring of vision and was diagnosed to have right branch retinal vein occlusion (BRVO).

- Over next 4/12: progressed to right central retinal venous occlusion (CRVO) and also developed left CRVO soon thereafter.

- Intravitreal Ranibizumab injections and Timolol-Dorzolamide eye drops were initiated for elevated IOP (OD $30 \mathrm{mmHg}$, OS 26 $\mathrm{mmHg}$ ).

\section{Further course}

- During an admission for intercurrent sepsis detected to have persistent hypokalaemia and abnormal thyroid function tests.

- Endocrine referral 8/52 later: severe fatigue, $15 \mathrm{~kg}$ weight loss, poor sleep and poor memory, proximal muscle weakness but no striae, bruising or signs of fat deposition and redistribution.

- Biochemistry: confirmed Cushing's syndrome (table 2) with suppression of gonadal, thyroid and GH axes (table 1).

- MRI: A 8x8x12mm pituitary adenoma (picture 1 )

- $>50 \%$ suppression of plasma cortisol after high-dose dexamethasone administration

- Diagnosis: Pituitary dependent Cushing's disease.

- Acute presentation with rapid weight loss and hypokalaemia raised suspicion of ectopic ACTH overproduction and he was referred to the regional pituitary centre for CRH-IPSS for confirmation.

- Rapid deterioration with further $10 \mathrm{~kg}$ weight loss, recurrent falls and cognitive decline eventually leading to severe acute psychosis.

- ITU admission and IV Etomidate f/b emergency transsphenoidal hypophysectomy without further confirmatory tests.

- Post-surgery: undetectable ACTH, dramatic clinical improvement, rapid recovery of all pituitary axes besides adrenal.

- Vision: OS- complete recovery, OD- limited to hand movements.

Table 1: Laboratory investigations on presentation and post-surgery

\begin{tabular}{|l|c|c|c|} 
& $\begin{array}{c}\text { On } \\
\text { presentation }\end{array}$ & $\begin{array}{c}\text { Post- } \\
\text { surgery }\end{array}$ & $\begin{array}{c}\text { Reference } \\
\text { range }\end{array}$ \\
\hline Sodium (mmol/L) & $\mathbf{1 4 2}$ & $\mathbf{1 4 3}$ & $133-146$ \\
\hline Potassium (mmol/L) & $\mathbf{2 . 9}$ & $\mathbf{5 . 1}$ & $3.5-5.3$ \\
\hline HbA1c (mmol/mol) [\%] & $\mathbf{3 3}[\mathbf{5 . 2}]$ & $\mathbf{2 9}[\mathbf{4 . 8}]$ & $<48[<6.5]$ \\
\hline TSH (mU/L) & $\mathbf{0 . 3 5}$ & $\mathbf{1 . 5 5}$ & $0.35-4.9$ \\
\hline Free T4 (pmol/L) & $\mathbf{2 . 4}$ & $\mathbf{4 . 1}$ & $2.6-5.7$ \\
\hline Free T3 (pmol/L) & $\mathbf{8 . 9}$ & $\mathbf{1 2 . 0}$ & $9.0-19.0$ \\
\hline Testosterone (nmol/L) & $\mathbf{1 . 3}$ & $\mathbf{1 0 . 1}$ & $4.9-32.0$ \\
\hline LH (IU/L) & $\mathbf{0 . 5}$ & $\mathbf{3 . 1}$ & $0.6-12.0$ \\
\hline FSH (IU/L) & $\mathbf{0 . 8}$ & $\mathbf{7 . 1}$ & $1.0-11.9$ \\
\hline GH (ug/L) & $<\mathbf{0 . 1}$ & $\mathbf{0 . 2}$ & - \\
\hline IGF-1 (nmol/L) & $\mathbf{9 . 1}$ & $\mathbf{1 7 . 5}$ & $8.9-32$ \\
\hline Prolactin (m/U/L) & $\mathbf{2 9 3}$ & $\mathbf{2 3 8}$ & $58-419$ \\
\hline
\end{tabular}

Table 2: Investigations for hypercortisolism and its aetiology

\begin{tabular}{|l|c|c|}
\hline & Value & $\begin{array}{c}\text { Reference } \\
\text { range }\end{array}$ \\
\hline 9 am cortisol (nmol/L) & $\mathbf{7 5 6}$ & $102-535$ \\
\hline 24-hour urinary free cortisol (nmol/24hr) & 2563 & $<130.0$ \\
\hline $\begin{array}{l}\text { 9 am cortisol post-overnight 1 } \mathrm{mg} \\
\text { dexamethasone (nmol/L) }\end{array}$ & 656 & $<50$ \\
\hline \begin{tabular}{l} 
High-dose dexamethasone suppression test \\
\multicolumn{1}{|c|}{ Baseline cortisol (nmol/L) }
\end{tabular} & $\mathbf{8 6 1}$ & $102-535$ \\
\hline \multicolumn{1}{|c|}{48 -hour cortisol (nmol/L) } & 310 & - \\
\hline ACTH (baseline) (ng/L) & $\mathbf{2 1 4 . 6}$ & $7.2-63.3$ \\
\hline ACTH (48 hour) (ng/L) & 127.7 & - \\
\hline
\end{tabular}

Picture 1: MRI scan of pituitary gland showing left sided adenoma

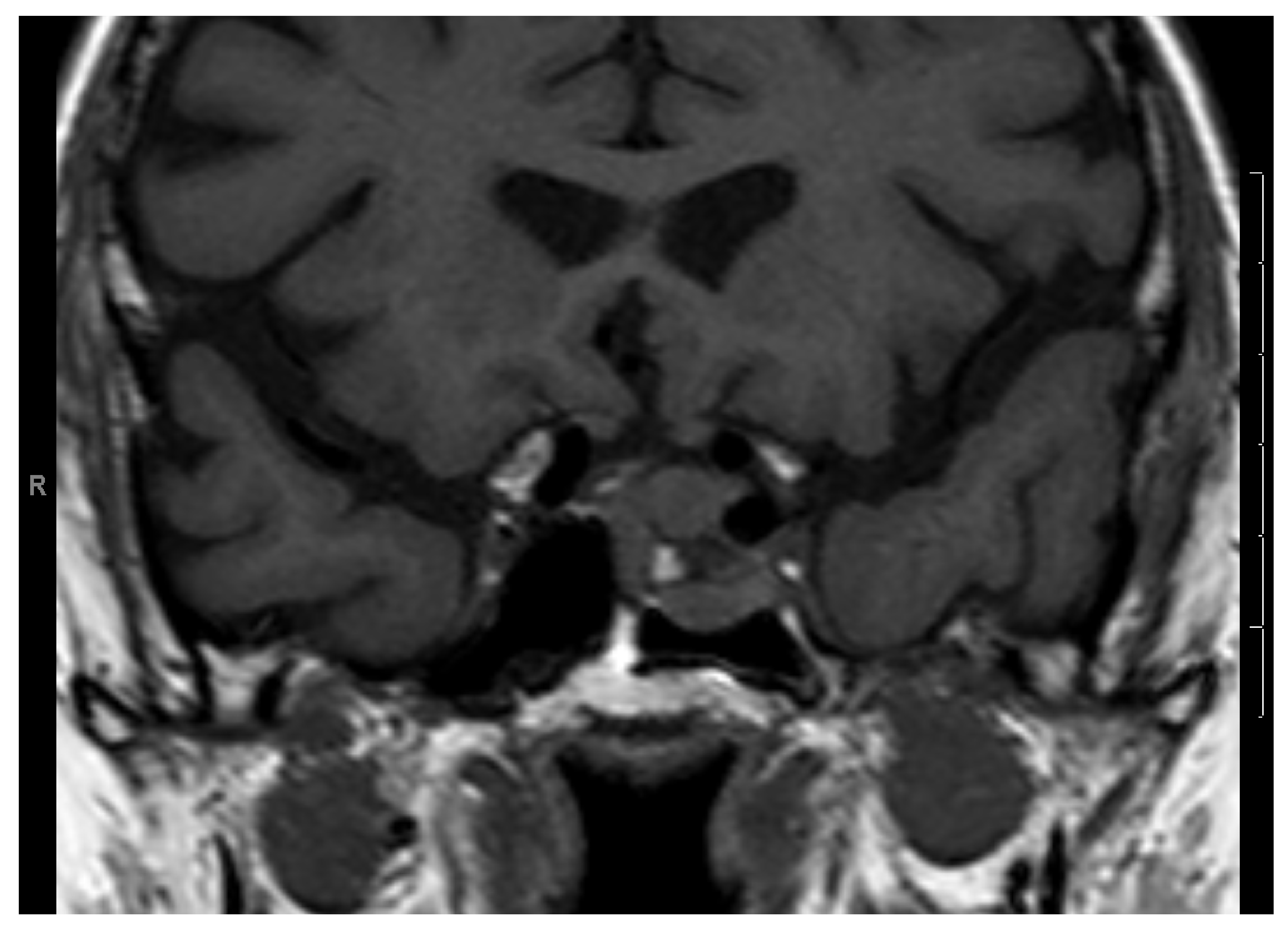

Discussion:

When presented with bilateral CRVO the patient did not manifest any other features of Cushing's syndrome and only 3 months later he had dramatic weight loss, muscle weakness and acute psychosis. Absence of hyperglycaemia and fat redistribution despite severe hypercortisolism was also noteworthy.

It is highly likely that bilateral CRVOs were related to the $>10$-fold higher risk of developing venous thromboembolism in Cushing's syndrome but it has not been reported in literature before. Several mechanisms have been proposed; aPTT is shortened as a result of higher levels of procoagulant factors like fibrinogen, factor VIII, and protein S. Clot-lysis time is also prolonged due to impaired fibrinolytic activity as a result of increase in PAI-1, TAFI and 2-AP [1]. Even though coagulation abnormalities seem to improve one year following successful surgery, they do not normalize [2] and we are not aware of any large scale study which can form the basis of thromboprophylaxis recommendation.

Acute severe psychosis in Cushing's syndrome is extremely rare. Treatment is generally with intravenous Etomidate infusion to block cortisol synthesis and oral Mifepristone which competitively binds the glucocorticoid receptors [3] along with early localization and surgical resection of the tumour [4] and this formed the basis of early intervention in our patient.

\section{References:}

van der Pas R et al. Hypercoagulability in Cushing's syndrome: prevalence, pathogenesis and treatment Clin Endocrinol (Oxf). 2013 Apr:78(4):481-8.

Manetti L et al. Changes in coagulation indexes and occurrence of venous thromboembolism in patients with Cushing's syndrome: results from a prospective study before and after surgery. Eur J Endocrinol. 2010 Nov;163(5):783-91. Bilgin YM et al. Treatment of severe psychosis due to ectopic Cushing's syndrome. J Endocrinol Invest. 2007 Oct 11:30(9):776-9.

Baba M et al. Severe psychosis due to Cushing's syndrome in a patient with a carcinoid tumour in the lung: a case report and review of the current management. World J Surg Oncol. 2015 Apr 30;13:165. 